\title{
Admissions due to pneumonia and biomass burning: a spatial approach
}

\author{
Internações por pneumonias e queimadas: uma abordagem espacial
}

\author{
Luiz Fernando C. Nascimento', Andréa Paula Peneluppi de Medeiros²
}

\section{Resumo}

Objetivo: Identificar padrões espaciais para taxas de internação por pneumonia em crianças e para focos de queimada no estado do Mato Grosso.

Metodologia: Foi realizado um estudo ecológico e exploratório com dados de internação de pneumonia em criança de 0 a 4 anos e de focos de queimada para o estado do Mato Grosso relativos aos anos de 2008 e 2009. Foram criadas taxas de internação, construídos mapas temáticos para essas taxas e para focos de queimada e obtidos os índice de Moran global e estimador de Kernel para as taxas e focos de queimada. Os dados foram analisados pelo programa TerraView 3.3.1.

Resultados: Foram 15.689 internações (variando de zero a 2.315) e 161.785 focos de queimadas (variando de sete a 6.454). A taxa média de internação por 1.000 habitantes foi de 2,89 [desvio padrão $=5,18$ ] e de queimadas por 1.000 habitantes foi de 152,81 (desvio padrão $=199,91$ ). 0 índice de Moran global para o número de internações foi de 0,02 $(p=0,26)$, para a taxa de internação foi de 0,02 ( $p$-valor $=0,21)$, e de $0,31$ ( $p$-valor $<0,01)$ para focos de queimadas. Foi possível identificar quatro municípios com elevadas taxas de internação por pneumonia e identificar duas regiões com altas densidades de internação. Ficou evidente um adensamento de focos de queimadas no chamado arco de desmatamento.

Conclusões: Este estudo permitiu identificar municípios que necessitam de intervenção para diminuírem as taxas de internação e focos de queimada no estado do Mato Grosso.

J Pediatr (Rio J). 2012;88(2):177-83: Pneumonia, sistema de informação geográfica, incêndios florestais, estudos ecológicos.

\begin{abstract}
Objective: To identify spatial patterns in rates of admission for pneumonia among children and relate them to the number of fires reported in the state of Mato Grosso, Brazil.

Methods: We conducted an ecological and exploratory study of data from the state of Mato Grosso for 2008 and 2009 on hospital admissions of children aged 0 to 4 years due to pneumonia and on fires in the same period. Admission rates were calculated and choropleth maps were plotted for rates and for fire outbreaks, Moran's I was calculated and the kernel estimator used to identify "hotspots." Data were analyzed using TerraView 3.3.1.
\end{abstract}

Results: Fifteen thousand six hundred eighty-nine children were hospitalized (range zero to 2,315), and there were 161,785 fires (range 7 to 6,454 ). The average rate of admissions per 1,000 inhabitants was 2.89 (standard deviation $[\mathrm{SD}]=5.18$ ) and the number of fires per 1,000 inhabitants was $152.81(S D=199.91)$. Moran's I for the overall number of admissions was $I=0.02(p=0.26)$, the index for rate of admission was $I=0.02(p=0.21)$ and the index for the number of fires was $I=0.31$ $(p<0.01)$. It proved possible to identify four municipalities with elevated rates of admissions for pneumonia. It was also possible to identify two regions with high admission densities. A clustering of fires was evident along what is known as the "arc of deforestation."

Conclusions: This study identified municipalities in the state of Mato Grosso that require interventions to reduce rates of admission due to pneumonia and the number fires.

J Pediatr (Rio J). 2012;88(2):177-83: Pneumonia, geographic information system, fires, biomass burning, ecological studies.

\section{Introdução}

No Brasil, as doenças respiratórias são responsáveis por $10,6 \%$ do total de mortes. Em 2008, o óbito por essa causa entre as crianças menores de 1 ano foi de 5,6\% e entre 1 e 4 anos foi de $17,0 \%$, ambas ocupando a terceira causa de óbito nas respectivas faixas etárias ${ }^{1}$. As doenças respiratórias

agudas e crônicas ocupam posição de destaque e encontramse entre as principais causas de internação no Sistema Único de Saúde (SUS). Em 2009, essas doenças ocuparam o segundo lugar em frequência, sendo responsáveis por 13,8\% de todas as internações do sistema ${ }^{1}$.

1. Doutor, Saúde Pública, Universidade de São Paulo (USP), São Paulo, SP. Departamento de Medicina, Universidade de Taubaté, Taubaté, SP. Programa de Pós-Graduação em Ciências Ambientais, Universidade de Taubaté, Taubaté, SP.

2. Doutora em Ciências, USP, São Paulo, SP. Departamento de Medicina, Universidade de Taubaté, Taubaté, SP.

Não foram declarados conflitos de interesse associados à publicação deste artigo.

Apoio financeiro: FAPESP Projeto Temático, Combustão de Biomassa de Florestas Tropicais. Processo 2008/04490-4.

Como citar este artigo: Nascimento LF, de Medeiros AP. Admissions due to pneumonia and biomass burning: a spatial approach. J Pediatr (Rio J). 2012;88(2):177-83

Artigo submetido em 26.07.11, aceito em 19.10.11.

http://dx.doi.org/10.2223/JPED.2161 
Dentre os fatores de risco para internação hospitalar por doenças respiratórias, está a exposição aos poluentes ambientais ${ }^{2-9}$. A discussão quanto à relevância dos determinantes ambientais na promoção à saúde e na prevenção de enfermidades é de fundamental importância na literatura. A exposição aos poluentes atmosféricos é reconhecida como um importante fator de risco para a ocorrência das internações hospitalares por pneumonia em crianças ${ }^{6,9}$. Internacionalmente, os efeitos da poluição atmosférica advinda da queima de florestas têm sido analisados principalmente na América do Norte e na Ásia10,11. No Brasil, o estado de São Paulo tem produzido estudos relativos à associação da poluição atmosférica, derivada de fontes móveis e da queima de cana-de-açúcar, e o aumento de internações por doenças respiratórias em crianças $3,6,12$. As fontes estacionárias e grandes frotas de veículos concentram-se nas áreas metropolitanas localizadas principalmente na região sudeste do país, enquanto a queima de biomassa ocorre em maior extensão e intensidade na Amazônia Legal. Situada ao norte, essa região concentra mais de $85 \%$ das queimadas que ocorrem no Brasil durante o período de estiagem das chuvas na região. O estado do Mato Grosso tem registrado anualmente o maior número de focos de queimadas e possui a maior área desmatada da Amazônia Legal desde o início dos anos $90^{13}$.

Os cenários de saúde desenhados pelas técnicas georreferenciadas agregam dados sócio-econômicos, ambientais e estruturais da saúde ao componente geográfico da relação de vizinhança entre os lugares. Nesse contexto, alguns autores ${ }^{14}$ identificaram áreas de risco para pneumonia em Goiânia (GO). Em outro estudo sobre os padrões espaciais, as internações por pneumonia em menores de 1 ano de idade foram analisadas, identificando-se os municípios no Vale do Paraíba Paulista com prioridade para intervenção ${ }^{15}$.

Embora nas últimas décadas tenha ocorrido um declínio na mortalidade em crianças por pneumonia, esse agravo ainda é importante causa de internação e morte nos países em desenvolvimento ${ }^{16}$.

O conhecimento de como essa carga de doença varia geograficamente fornece pistas para a compreensão dos determinantes dessas doenças e discernimento para a gestão eficaz dos recursos de saúde e, consequentemente, para a melhoria da qualidade de vida da população ${ }^{12}$. O objetivo deste estudo é identificar padrões espaciais nas taxas de internação por pneumonia em crianças, segundo focos de queimadas, no estado de Mato Grosso.

\section{Métodos}

Trata-se de estudo ecológico, do tipo exploratório, com dados de internação por pneumonias em crianças de 0 a 4 anos obtidos do Departamento de Informática do SUS (DATASUS) e de focos de queimadas relativos ao estado do Mato Grosso no período compreendido entre 01/01/2008 e 31/12/200917.

Os dados obtidos do DATASUS foram contabilizados no total dos 2 anos da mesma forma que os focos de queimadas, não sendo separados pelas estações chuvosa ou seca. Foram obtidos os dados da população para a criação de taxas de internação por 1.000 habitantes.

A planilha com os dados de população, número de internação por pneumonia e focos de queimada foi importada pelo programa TerraView 3.3.1, de acesso público e disponível para a análise espacial ${ }^{18}$.

O estado do Mato Grosso situa-se na Região CentroOeste do Brasil e compreende 141 municípios distribuídos numa área de aproximados $900 \mathrm{mil} \mathrm{km}^{2}$, com população de pouco menos de 3 milhões de habitantes. Situa-se entre os paralelos $9^{\circ}$ e $17^{\circ}$ Sul e longitude $50^{\circ}$ e $60^{\circ}$ Oeste. Tem uma densidade populacional de 2,6 habitantes $/ \mathrm{km}^{2}$.

Os dados de pneumonias se referem às internações por município, segundo o local de residência e de acordo com o diagnóstico relatado no portal do DATASUS ${ }^{19}$.

A malha digital dos municípios foi obtida do Instituto Brasileiro de Geografia e Estatística (IBGE) ${ }^{20}$. A unidade de análise foi o município. Foram criadas taxas de internação por 1.000 habitantes e estimado o índice de Moran $\left(\mathrm{I}_{\mathrm{M}}\right.$ ) global.

O $I_{M}$ é uma medida global da autocorrelação espacial, que indica o grau de associação espacial no conjunto de informações a partir do produto em relação à média. Seu valor pertence ao intervalo $(-1 ; 1)$. Ele testa se as áreas vizinhas apresentam maior semelhança quanto ao indicador estudado do que o esperado se fosse um padrão aleatório; quanto mais próximo do valor 1 , maior a semelhança entre os vizinhos (correlação positiva), e quanto mais próximo de -1 , indica diferenças das distribuições espacial entre os vizinhos (correlação negativa) ${ }^{21}$.

A distribuição das densidades de taxas de internação foi apresentada pela manchas quentes (hot spot) fornecidas pelo estimador de Kernel; esse estimador assumiu que todos os casos se localizaram no ponto central do município (centroide de cada município); e, para essa análise, foi utilizada uma grade de 200 colunas, com algoritmo de função quártica, raio adaptativo e cálculo de densidade com 10 fatias e precisão quatro.

Foi construído o mapa de Moran, que permite identificar municípios com altos valores (de taxa de internação e de focos de queimadas) circundados por outros com altos valores (situação alto-alto) e municípios com baixos valores (de taxa de internação e de focos de queimadas) circundados por outros com baixos valores (situação baixo-baixo); dessa forma, o mapa de Moran identificou municípios com alta prioridade de intervenção e outros com baixa prioridade de intervenção.

As taxas de internação e a distribuição dos focos de queimadas são apresentadas sob a forma de mapas temáticos (coropletes).

Foi estimado o coeficiente de correlação de Pearson para as variáveis: casos de pneumonia, taxa de internação e focos de queimadas.

O projeto foi submetido ao Comitê de Ética em Pesquisa da Universidade de Taubaté e aprovado sob o número $314 / 04$ 


\section{Resultados}

No período de estudo, foram internadas 15.689 crianças nos 141 municípios do estado do Mato Grosso (esse número variou entre zero e 2.315 internações), e foram registrados 161.785 focos de queimadas, com mínimo de sete e máximo de 6.454 por município. A taxa média de internação por 1.000 habitantes foi de 2,89 [desvio padrão $(D P)=5,18]$ e de queimadas por 1.000 habitantes foi de $152,81(D P=199,91)$. O I $I_{M}$ global para o número de internações foi de $0,02(p=0,26)$, para a taxa de internação foi de 0,02 ( $p$-valor $=0,21$ ) e para o número de focos de queimadas foi de 0,31 ( $p$-valor $<0,01$ ). Esses valores indicam que tanto as internações como as taxas de internações por pneumonias nos municípios de Mato Grosso não apresentam correlação espacial, isto é, os valores se distribuem aleatoriamente sem que esses municípios formem o chamado aglomerado espacial; para as queimadas, o $I_{M}$ apresentou significância estatística, evidenciando que esses eventos formam aglomerados, isto é, há grupos de municípios vizinhos com padrões semelhantes de número de focos de queimada.

Os mapas de Moran revelaram municípios onde uma intervenção se faz necessária, tanto para diminuir as taxas de internação como os focos de queimadas (Figuras 1a e 1b). São quatro municípios que necessitam de uma intervenção em virtude das altas taxas de internação: três estão localizados na região leste, próximo à divisa com o estado de Goiás, com populações entre 5 e 20 mil habitantes; outro se localiza na região oeste, próximo à divisa com Rondônia, e tem cerca de 20 mil habitantes. Dez municípios apresentam taxas

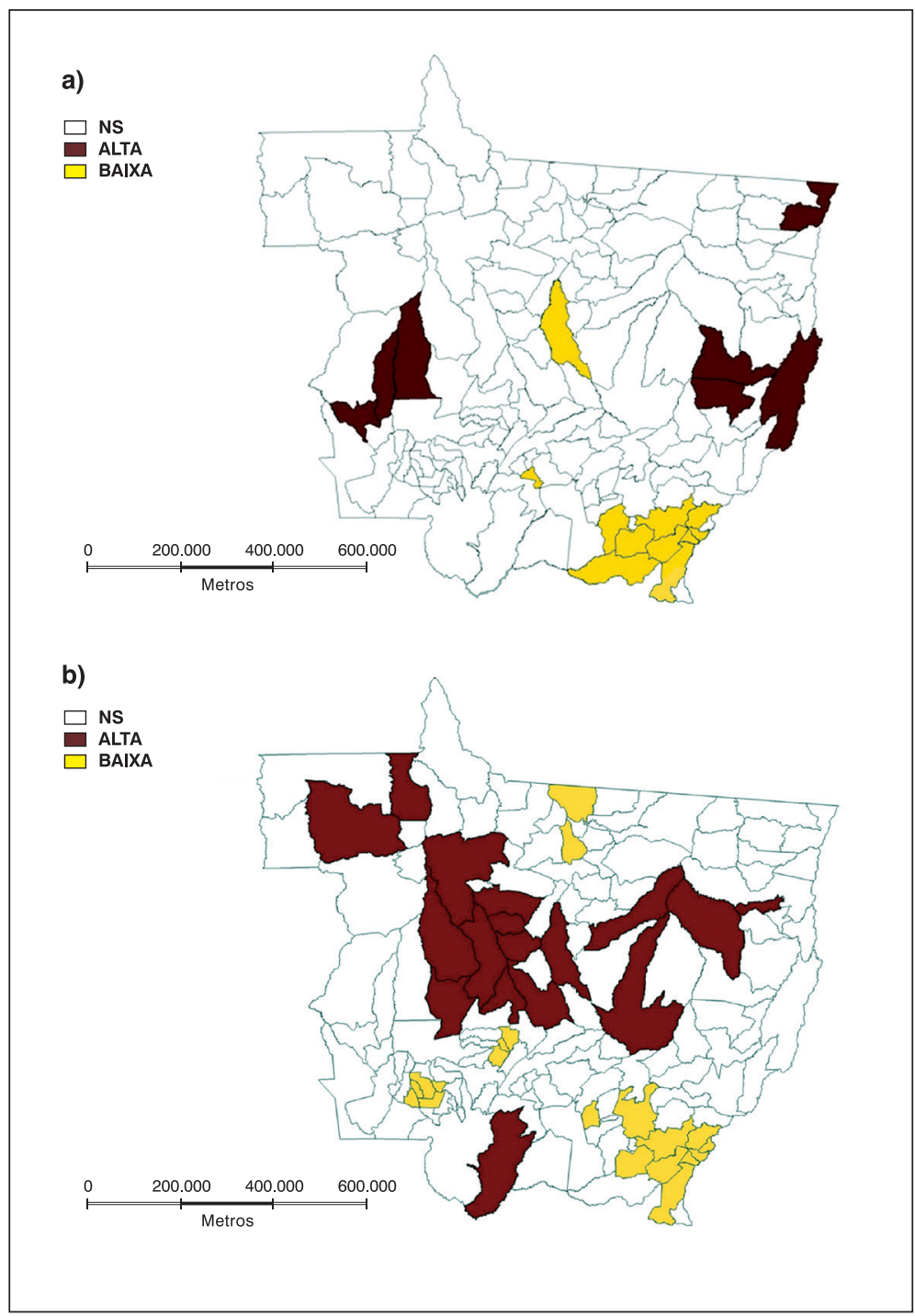

NS = não significante

Figura 1 - Municípios com alta e baixa prioridades de intervenção para taxas de internação por pneumonias (a) e focos de queimadas (b), Mato Grosso, Brasil, 2008-2009 
abaixo da média: um deles se localiza na região central do estado, com cerca de 60 mil habitantes; nove se localizam na região sudeste, próximos às divisas com os estados de Goiás e Mato Grosso do Sul.

Para as queimadas, os municípios com alta prioridade de intervenção compõem o chamado arco de desmatamento, e os com baixa prioridade se concentram na região sudeste, como aquele padrão mostrado para as internações por pneumonia.

O estimador de Kernel mostrou um adensamento (hot spot) de maiores taxas de internação localizado na divisa de Mato Grosso com Goiás, e outro, de menor intensida- de, na região sudoeste do estado em direção a Rondônia (Figura 2a). Para a distribuição de focos de queimadas, a distribuição espacial é significativa, e os municípios com maiores números de eventos formam um arco, também chamado arco de desmatamento (Figura 2b). Os coeficientes de correlação de Pearson estão na Tabela 1.

\section{Discussão}

Este estudo procurou identificar municípios do estado do Mato Grosso com altas taxas de internação por pneumonia em crianças e também municípios com elevado número de focos de queimadas nos anos de 2008 e 2009.

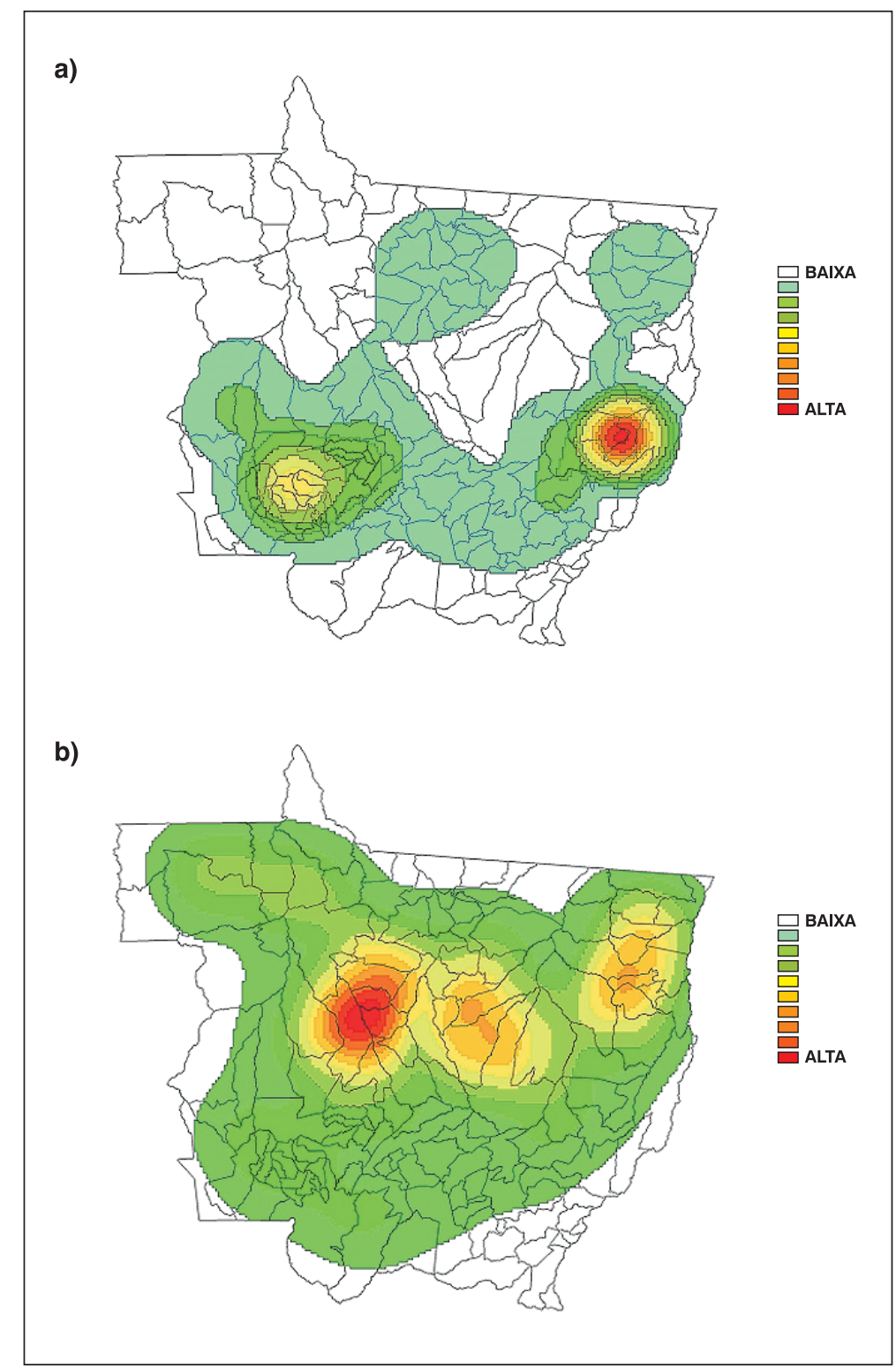

Figura 2 - Mapas temáticos para as densidades de taxas de internação por pneumonias (a) e focos de queimadas (b), Mato Grosso, Brasil, 2008-2009 
Tabela 1 - Coeficientes de correlação de Pearson para as variáveis de estudo, Mato Grosso, Brasil, 2008-2009

\begin{tabular}{|c|c|c|c|c|}
\hline & População & Casos & Queimadas & Taxa de internação \\
\hline População & 1 & & & \\
\hline Casos & $0,66^{*}$ & 1 & & \\
\hline Queimadas & $-0,29$ & 0,07 & 1 & \\
\hline Taxa de internação & $-0,15$ & $0,31^{*}$ & 0,05 & 1 \\
\hline
\end{tabular}

${ }^{*} p<0,01$.

A pneumonia é uma doença com alta taxa de letalidade, haja vista que, no período de estudo, foram 150 óbitos no estado do Mato Grosso tendo como causa básica essa doença, num total de 1.956 óbitos na faixa etária de 0 a 4 anos, representando $7,7 \%$ do total de óbitos ${ }^{22}$. É importante salientar que, na faixa etária até 1 ano, doenças incluídas nos capítulos XVI (algumas afecções originadas no período perinatal) e XVII (malformações congênitas, deformidades e anomalias cromossômicas) têm reconhecida contribuição para os óbitos. Por outro lado, o custo financeiro das internações por pneumonia, neste período, para o estado do Mato Grosso e nesta faixa etária, foi de R $\$ 10$ milhões (1 US\$ $~ R \$ 1,80$ ), representando $25 \%$ do total de gastos com todas as demais internações nessa mesma faixa etária ${ }^{19}$.

A queimada, que pode ser de origem natural ou, mais frequentemente na Região Centro-Oeste, de origem antrópica, libera monóxido e dióxido de carbono, metano, material particulado, óxidos de nitrogênio e ozônio; este último poluente atmosférico é gerado pela ação da luz solar sobre os compostos nitrogenados ${ }^{23}$. Além desses poluentes, as queimadas liberam aldeídos, hidrocarbonetos, ácidos inorgânicos e hidrocarbonetos aromáticos policíclicos que podem produzir manifestações clínicas importantes em adultos e crianças $^{24}$.

As queimadas representam mais de $70 \%$ das emissões brasileiras de carbono 25 . O estado do Mato Grosso é um dos que apresentam maiores índices de focos de queimada. Nos anos estudados, de 2008 e 2009, foram apontados mais de 160 mil focos. Ao contrário de estudo realizado por Ignotti et al.13, não foram selecionados municípios com altas taxas de internação por doenças respiratórias ou municípios com mais de 25 mil habitantes. Todos os 141 municípios do estado do Mato Grosso foram incluídos no estudo.

Uma vantagem do presente estudo é a utilização do geoprocessamento, que permite a integração de dados ambientais com dados de saúde, melhorando a caracterização e quantificação da exposição, seus possíveis determinantes e os agravos à saúde. Essa metodologia procura identificar, na estrutura espacial e nas relações sociais, as associações plausíveis com os processos de adoecimento e morte nas coletividades. As cidades não estão isoladas no espaço, elas estão interligadas a outros centros urbanos de maior ou de menor nível e a áreas rurais com os quais estabelecem relações de troca. O grau de conectividade e importância de uma cidade, em geral, tem um efeito nos processos de difusão de doenças, ou seja, as mais conectadas são atingidas com maior rapidez ${ }^{26}$.

A distribuição espacial das taxas de internação não apresentou autocorrelação significativa. Por outro lado, os focos de queimadas seguiram um padrão espacial com significância estatística; sua distribuição geográfica segue um caminho de noroeste para nordeste do estado numa forma de arco, com a concavidade para cima na região denominada "arco de desmatamento".

Quando a taxa de internação foi analisada pelo estimador de Kernel, observou-se uma região com maior adensamento de municípios com taxas maiores (hot spot) na região leste do estado, na divisa com o estado de Goiás, e outra, menos densa, na região oeste, na divisa com o estado de Rondônia; esse comportamento foi semelhante ao encontrado para internações por asma25.

Quando se analisa o estimador de Kernel para os focos de queimadas, é notável a identificação do arco de desmatamento. Nessa região, as queimadas são realizadas para a ocupação do solo, tanto para a expansão das fronteiras agrícolas como para a pecuária de corte. No entanto, as regiões com maiores densidades de focos se situam ao norte das regiões com maiores densidades para as taxas de internação.

Essa distribuição pode ser explicada pelo fato de os poluentes produzidos pelas queimadas serem levados para a troposfera ( 3 a $10 \mathrm{~km}$ de altura) por movimentos convectivos gerados pelas altas temperaturas que ocorrem na fase de chamas da combustão; em decorrência de ventos, a fumaça com seus componentes podem atingir regiões afastadas dos focos 23 . 
Um estudo realizado em Piracicaba (SP), município onde predomina a cultura de cana-de-açúcar, mostrou que a queima da palha da cana aumenta o risco de doenças do aparelho respiratório em crianças e idosos ${ }^{3}$. A palha da canade-açúcar queimada em meses secos gerou três vezes mais material particulado (PM10) além do dobro de black carbon $(B C)$ e metais como alumínio (Al), enxofre $(S)$, potássio $(K)$ e silício ( $\mathrm{Si}$ ). Houve um aumento ao redor de $25 \%$ nas internações por problemas respiratórios em crianças, atribuído à presença de $\mathrm{PM} 10$ e $\mathrm{BC}$ decorrentes da queima da palha de cana-de-açúcar.

No estudo aqui apresentado, os dados não foram analisados separadamente de acordo com as estações seca e chuvosa. Os focos de queimadas são registrados em número muito maior nos meses secos (maio a outubro), correspondendo a $90 \%$ das ocorrências ${ }^{17}$.

O mapa de Moran identificou seis municípios que necessitam de uma intervenção em virtude das altas taxas de internação; quatro estão localizados na região leste, próximos à divisa com o estado de Goiás, com populações entre 5 e 20 mil habitantes; outros dois se localizam na região oeste, próximos à divisa com Rondônia e à Tangará da Serra. Esse fato pode sugerir que esteja havendo uma demanda maior em Tangará da Serra, que não é advinda desse município, mas de municípios vizinhos; uma possibilidade é pelo fato de Tangará da Serra ser um polo regional, com boa estrutura de atendimento médico. Muitos munícipes de cidades vizinhas informam um endereço fictício em Tangará da Serra para poder usar seus serviços de saúde.

Os municípios que se classificam nesse grupo de alta prioridade devem estabelecer o objetivo de diminuir essas taxas para valores de seus vizinhos com média ou baixa prioridade. Por outro lado, essas taxas podem estar associadas ao número de focos de queimadas ocorridos a $400 \mathrm{~km}$ a noroeste e cujos componentes tenham sido trazidos por ventos.

A abordagem espacial tornou possível a identificação de 11 municípios com taxas de internação abaixo da média; nove deles se localizam na região sudeste, próximos às divisas com os estados de Goiás e Mato Grosso do Sul. São municípios com baixa prioridade de intervenção. Estão localizados a mais de $600 \mathrm{~km}$ da região com maiores densidades de focos. Por outro lado, aquele que se localiza na região central do estado tem cerca de 60 mil habitantes e é cortado pela rodovia Cuiabá-Santarém. Tem um hospital geral com 233 leitos. Essas características talvez permitam um melhor acesso aos serviços de saúde e expliquem essas baixas taxas de internação. No estudo de Ignotti et al. ${ }^{13}$, esse município apresentou um baixo escore para internação em municípios com mais de 25 mil habitantes. Outro município com baixas taxas se localiza na região metropolitana de Cuiabá; sua situação geográfica explica essas taxas baixas, haja vista a possível facilidade de acesso a serviços de saúde, bem como a disponibilidade de profissionais da saúde nesse município.

Na região sudeste do estado, existe um polo regional importante que é Rondonópolis, cortada pela rodovia BR-364. Possivelmente, esses fatos permitam um melhor acesso aos serviços de saúde, e a região deve dispor de especialistas, o que permite diminuir as taxas de internação. Os municípios localizados nessa região têm baixas taxas de internação.

Por outro lado, na região denominada arco de desmatamento, era de se esperar um número maior de internações. Uma possível explicação seria a dificuldade para o atendimento médico, seja pela falta de profissionais, seja pelas distâncias a serem percorridas entre a ocorrência do fato e a cidade. Outra possibilidade seria uma subnotificação dos casos que seriam codificados sob outro diagnóstico.

Outro ponto forte do estudo foi identificar os municípios com altas taxas de internação por pneumonias, pois permitirá a diminuição dos óbitos nessa faixa etária por essa doença, declínio que vem ocorrendo, também, na Região Centro-Oeste27.

Estudos ecológicos costumam ter limitações. Podemos citar, entre elas, o fato de se trabalhar com dados secundários. Neste estudo, a fonte de dados foi o DATASUS, que os tem disponibilizado, e vários estudos têm sido realizados com estes $6,12,25$. Há que se considerar erros no diagnóstico de internação, sendo que este estudo não confirmou o diagnóstico de pneumonia como realizado por Andrade et al. ${ }^{14}$ em Goiânia. Outras limitações seriam uma superestimação diagnóstica e também o fornecimento incorreto da residência da criança internada, fazendo com que aumentassem as taxas de internação.

Os resultados apresentados representam uma aproximação quantitativa do impacto da poluição atmosférica na saúde da população. É importante salientar que o desfecho aqui estudado, hospitalização, é apenas um dos muitos efeitos causados pela poluição do ar. Este estudo utiliza dados secundários para o cálculo dos coeficientes para hospitalizações decorrentes do aumento do nível de poluição atmosférica observada.

Esses dados são provenientes de fontes estáveis e bastante confiáveis, sendo utilizados amplamente em trabalhos técnicos e científicos. É importante ressaltar que os registros são referentes a hospitalizações ocorridas na rede pública de saúde. Dessa forma, os resultados aqui apresentados refletem os efeitos da poluição do ar na parcela da população que utiliza esse serviço, que corresponde à maior parte da população do país. Por outro lado, as informações do Sistema de Informações Hospitalares do SUS utilizadas neste estudo são produzidas com objetivos contábeis, e não estritamente para estudos epidemiológicos, podendo, assim, apresentar algum grau de inacurácia. Pode ter havido ainda algum grau de subestimação da frequência de determinados agravos, tendo em vista o perfil tecnológico da rede de assistência, além de erros na codificação dos diagnósticos. Um outro problema presente nessa base de dados é a possibilidade da dupla contagem de um mesmo paciente, uma vez que o sistema não identifica reinternações.

Por outro lado, há que se lembrar de que se trata de um estudo ecológico em que não se dispõe de informações individuais sobre exposição e doença. Por exemplo, não se pode afirmar com certeza que aqueles indivíduos que foram hospitalizados eram os mais expostos aos níveis de poluição do ar. Todavia, como a exposição é medida de maneira ecológica, assume-se que uma variação no nível médio de 
poluição do ar num determinado dia no município reflita também uma variação na exposição média de cada indivíduo residente nele.

Este estudo mostrou municípios com altas taxas de internação que não se encontram na região onde ocorre maior número de focos de queimadas, e sim distantes vários quilômetros, sugerindo que os produtos resultantes dessas queimadas possam ser transportados por correntes de vento, que costumam ter direção noroeste-sudeste, causando problemas de saúde em populações distantes dos eventos.

\section{Referências}

1. Brasil, Ministério da Saúde. Cadernos de Informação de Saúde. Departamento de Informática do SUS - DATASUS. http://tabnet. datasus.gov.br/tabdata/cadernos/cadernosmap.htm. Acesso: 25/02/2011.

2. Farhat SC, Paulo RL, Shimoda TM, Conceição GM, Lin CA, Braga $A L$, et al. Effect of air pollution on pediatric respiratory emergency room visits and hospital admissions. Braz J Med Biol Res. 2005;38:227-35.

3. Cançado JE, Saldiva PH, Pereira LA, Lara LB, Artaxo P, Martinelli $L A$, et al. The impact of sugar cane-burning emissions on the respiratory system of children and the elderly. Environ Health Perspect. 2006;114:725-9.

4. Schwartz J. Air pollution and children's health. Pediatrics. 2004; 113:1037-43.

5. Cançado JE, Braga A, Pereira LA, Arbex MA, Saldiva PH, Santos U de P. Clinical repercussions of exposure to atmospheric pollution. J Bras Pneumol. 2006;32:S5-11.

6. Nascimento LF, Pereira LA, Braga AL, Módolo MC, Carvalho JA Jr. Effects of air pollution on children's health in a city in Southeastern Brazil. Rev Saude Publica. 2006;40:77-82.

7. Ignotti $E$, Hacon $S$ de $S$, Junger $W L$, Mourão $D$, Longo $K$, Freitas $S$, et al. Air pollution and hospital admissions for respiratory diseases in the subequatorial Amazon: a time series approach. Cad Saude Publica. 2010;26:747-61.

8. Pope CA 3rd, Dockery DW. Health effects of fine particulate air pollution: lines that connect. J Air Waste Manag Assoc. 2006;56:709-42.

9. Braga AL, Saldiva PH, Pereira LA, Menezes JJ, Conceição GM, Lin CA, et al. Health effects of air pollution exposure on children and adolescents in São Paulo, Brazil. Pediatr Pulmonol. 2001;31:106-13.

10. Tan WC, Qiu D, Liam BL, Ng TP, Lee SH, van Eeden SF, et al. The human bone marrow response to acute air pollution caused by forest fires. Am J Respir Crit Care Med. 2000;161:1213-7.

11. Sapkota A, Symons JM, Kleissl J, Wang L, Parlange MB, Ondov J, et al. Impact of the 2002 Canadian forest fires on particulate matter air quality in Baltimore city. Environ Sci Technol. 2005;39:24-32.

12. Gouveia N, de Freitas CU, Martins LC, Marcilio IO. Hospitalizações por causas respiratórias e cardiovasculares associadas à contaminação atmosférica no município de São Paulo, Brasil. Cad Saude Publica. 2006;22:2669-77.
13. Ignotti $E$, Hacon SS, Silva AM, Junger WL, Castro HA. Efeitos das queimadas na Amazônia: método de seleção dos municípios segundo indicadores de saúde. Rev Bras Epidemiol. 2007; 10:453-64.

14. Andrade AL, Silva SA, Martelli CM, Oliveira RM, Morais Neto OL, Siqueira Júnior JB, et al. Population-based surveillance of pediatric pneumonia: use of spatial analysis in an urban area of Central Brazil. Cad Saude Publica. 2004;20:411-21.

15. Mukai A de O, Alves K de S, Nascimento LF. Spatial analysis of hospitalizations for pneumonia in the Vale do Paraíba region of Brazil. J Bras Pneumol. 2009;35:753-8.

16. Williams BG, Gouws E, Boschi-Pinto C, Bryce J, Dye C. Estimates of world-wide distribution of child deaths from acute respiratory infections. Lancet Infect Dis. 2002;2:25-32.

17. Brasil, Ministério da Ciência e Tecnologia, Instituto Nacional de Pesquisas Espaciais. Queimadas: monitoramento de focos. http:// www.dpi.inpe.br/proarco/bdqueimadas/. Acesso: 06/06/2011.

18. Brasil, Ministério da Ciência e Tecnologia, Instituto Nacional de Pesquisas Espaciais. Projeto TerraView. http://www.dpi.inpe.br/ terraview/index.php. Acesso: 04/06/2010.

19. Brasil, Ministério da Saúde, DATASUS: Informações de saúde. Morbidade Hospitalar do SUS - por local de residência - Mato Grosso. http://tabnet.datasus.gov.br/cgi/deftohtm.exe?sih/cnv/ nrmt.def. Acesso: 15/02/2011.

20. Brasil, Instituto Brasileiro de Geografia e Estatística. Índice de pastas. ftp://geoftp.ibge.gov.br/mapas/. Acesso: 06/06/2011.

21. Brasil, Ministério da Ciência e Tecnologia, Instituto Nacional de Pesquisas Espaciais. Análise Espacial de Dados Geográficos. http:// www.dpi.inpe.br/gilberto/livro/analise/. Acesso: 23/09/2011.

22. Brasil, Ministério da Saúde, DATASUS: Informações de saúde. Mortalidade - Mato Grosso. http://tabnet.datasus.gov.br/cgi/ tabcgi.exe?sim/cnv/obt10mt.def. Acesso: 23/09/2011.

23. Freitas SR, Longo KM, Dias MA, Dias PL. Emissões de queimadas em ecossistemas da América do Sul. Estud Av. 2005;19:167-85.

24. Arbex MA, Cançado JE, Pereira LA, Braga AL, Saldiva PH. Queima de biomassa e efeitos sobre a saúde. J Bras Pneumol. 2004;30:158-75.

25. Silva PR, Rosa AM, Hacon SS, Ignotti E. Hospitalization of children for asthma in the Brazilian Amazon: trend and spatial distribution. J Pediatr (Rio J). 2009;85:541-6.

26. Brasil, Ministério da Saúde, Secretaria de Vigilância em Saúde. Fundação Oswaldo Cruz; Simone M. Santos, Christovam Barcellos, orgs. Abordagens espaciais na saúde pública. Brasília: Ministério da Saúde; 2006. 136 p. (Série B. Textos Básicos de Saúde. Série Capacitação e Atualização em Geoprocessamento em Saúde; vol. 1).

27. Rodrigues FE, Tatto RB, Vauchinski L, Leães LM, Rodrigues MM, Rodrigues VB, et al. Pneumonia mortality in Brazilian children aged 4 years and younger. J Pediatr (Rio J). 2011;87:111-4.

Correspondência:

Luiz Fernando C. Nascimento

Rua Durval Rocha, 500 - Vila Paraíba

CEP 12515-710 - Guaratinguetá, SP

Tel.: (12) 3625.4271

E-mail: luiz.nascimento@unitau.br 